\section{Nomogram for the Computation of the Corrected Colour Value in 17-Ketosteroid Estimation by the Zimmerman Reaction}

\section{A. P. KENNY}

From the Biochemistry Department, Clinical Laboratories, Victoria Infirmary of Glasgow

(RECEIVED FOR PUBLICATION AUGUST 28, 1959)

The M.R.C. committee on clinical endocrinology (1951) recommends correcting the green extinction value in the determination of 17 -ketosteroids by the formula:

Corrected green extinction value

$$
=\begin{gathered}
\text { observed green } \\
\text { extinction value }
\end{gathered}-0.6 \times \begin{aligned}
& \text { observed violet } \\
& \text { extinction value }
\end{aligned}
$$

This formula, derived largely from the work of Talbot, Berman, and MacLachlan (1942), can easily be converted into the form $x=m_{1} y+m_{2} z$ which allows the construction of a simple nomogram (Feldman, 1935). The latter greatly accelerates calculation when several estimations are being done together, and reduces the risk of arithmetical error.

Thus if the observed extinction at $430 \mathrm{~m} \mu=\mathrm{E}_{430}=\mathrm{x}$, the observed extinction at $520 \mathrm{~m} \mu=E_{s 20}=y$, and the corrected extinction $=E$ (corr.) $=z$, the formula given above can be written: $y=0.73 z+0.6 x \ldots \ldots \ldots \ldots \ldots . .(2)$

To construct a nomogram of sufficient accuracy take a large sheet of 1 in. graph paper ( 20 in. by 15 in.), scaled in 1/10 inches, and draw three parallel vertical lines, 15 in. long and 5 in. apart and a base line along the bottom as shown in Fig. 1, and label the vertical lines $X, Y$, and $Z$, from left to right. On $X$ will be plotted the observed $E_{430}$ and on $Y$ the observed $E_{520}$, and $Z$ will then give $E_{\text {(corr.). The line }}$ $X$ is marked off in 2 in. divisions, each to correspond to 0.1 unit. It is subdivided further and at the point 0.730 unit a line is drawn parallel to the base to cut $\mathrm{Z}$ and this point is marked 0.600 unit and the length between the point and the base is divided into six equal divisions and subdivided further and also extended a little way upwards as shown.

In order to graduate line $\mathrm{Y}$, tie-lines are placed between point 0.300 on $X$ and 0.301 on $Z$ and where $Y$ is cut corresponds to 0.400 unit on this scale. Similarly by joining point 0.600 on $\mathrm{X}$ and 0.603 on $\mathrm{Z}$ the point 0.800 is found. Confirmation of the above points can be obtained by joining 0.500 on $X$ with 0.137 on $Z$ and 0.700 on $X$ with 0.521 on $Z$, after which $Y$ can be divided into eight equal divisions and further subdivided. The main $X, Y$, and $Z$ lines and their divisions are best traced on to a sheet of white Bristol board by pricking through the marks with a sharp needle after which the nomogram is drawn on the cardboard in Indian ink.

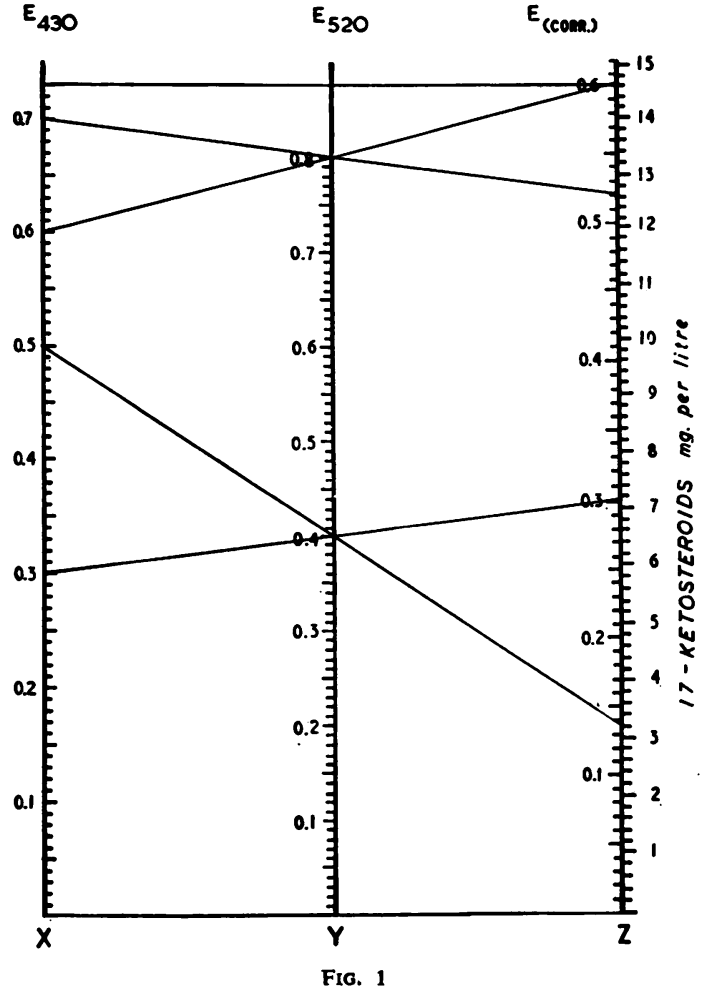

To evaluate the corrected reading the observed reading at $430 \mathrm{~m} \mu$ is joined with that obtained at $520 \mathrm{~m} \mu$, using a thin transparent ruler, and the required result is read off on line $Z$.

When a standard calibration for 17-ketosteroids determination has been made, a separate graph is plotted for the corrected reading against dehydroepiandrosterone expressed in terms of milligrams per litre of urine. The resultant line may not be strictly linear, but the interpolated values can be transferred to the nomogram and plotted alongside the corrected values on line $Z$, preferably in pencil to allow for periodic revision, so that a direct interpolation of 17-ketosteroids can be obtained. The nomogram shown above illustrates the inclusion of such a scale, which is shown to the right of line $Z$, and has been obtained using dehydroepiandrosterone as the standard substance and is based on the assumption that the residues from $100 \mathrm{ml}$. samples of urine have been taken up in $2 \mathrm{ml}$. ethanol before the colour development stage in the M.R.C. method (1951).

To obtain the urinary $17-$ ketosteroids in milligrams per 24 hours, the only calculation necessary is the multiplication of the result obtained from the nomogram by the 24-hour volume in litres.

\section{REFERENCES}

Feldman, W. M. (1935). Biomathematics, 2nd ed. Griffin, London. M.R.C. Committee on Clinical Endocrinology (1951). Lancet, 2, 585. Talbot, N. B., Berman, R. A., and MacLachlan, E. A. (1942). J. biol. Chem., 143, 211 . 